\title{
Borderline, avoidant, sadistic personality traits and emotion dysregulation predict different pathological skin picking subtypes in a community sample
}

This article was published in the following Dove Press journal:

Neuropsychiatric Disease and Treatment

I August 2016

Number of times this article has been viewed

\section{Andrea Pozza ${ }^{1,2}$ \\ Nicoletta Giaquinta ${ }^{2,3}$ \\ Davide Dèttore ${ }^{4}$}

'Department of Experimental and Clinical Medicine, University of

Florence, Florence, ${ }^{2}$ Miller Institute of Behavioral and Cognitive

Psychotherapy, Genoa, ${ }^{3}$ Centre of Cognitive and Behavioural Therapy (CTCC), Florence, ${ }^{4}$ Department of Health Sciences, University of Florence, Florence, Italy
Correspondence: Andrea Pozza Department of Experimental and Clinical Medicine, University of Florence, Largo Brambilla 3, 50I35, Florence; Miller Institute of Behavioral and Cognitive Psychotherapy, Corso Torino 19/2,

16129 Genoa, Italy

$\mathrm{Tel}+39105707062$

Fax +39108680904

Email andrea.pozza@unifi.it

\begin{abstract}
Pathological skin picking (SP) is a strongly impairing condition characterized by repetitive picking behaviors resulting in significant tissue damage and distress. Recent research suggested the presence of different subtypes of SP. No study has investigated which personality traits could be specifically associated with different subtypes. In a community sample $(\mathrm{N}=285$, $71.20 \%$ females, mean age $=34.98$ years, standard deviation $=15.91$ ), this cross-sectional study investigated which personality traits and emotion regulation deficits could predict specific SP subtypes. Participants completed the Milwaukee Inventory for the Dimensions of Adult Skin Picking (MIDAS), Millon Clinical Multiaxial Inventory-III personality scales (MCMI-III), and Difficulties in Emotion Regulation Scale (DERS) questionnaires. Higher scores on the MCMI-III borderline ( $\beta=0.28, t=4.88, P<0.001)$, MCMI-III avoidant scale $(\beta=0.18, t=2.59, P<0.01)$, and DERS difficulties engaging in goal-directed behavior $(\beta=0.19, t=3.27, P<0.01)$ predicted higher scores on the MIDAS automatic scale. Higher scores on the MCMI-III borderline $(\beta=0.30$, $t=5.23, P<0.001)$ and DERS difficulties engaging in goal-directed behavior $(\beta=0.15, t=2.52$, $P<0.05)$ and DERS limited access to emotion regulation strategies $(\beta=0.21, t=3.26, P<0.01)$ predicted higher scores on the MIDAS focused scale. Higher scores on the MCMI-III sadistic $(\beta=0.19, t=3.30, P<0.001)$ and DERS difficulties engaging in goal-directed behavior $(\beta=0.15$, $t=2.68, P<0.01)$ predicted higher scores on the MIDAS mixed scale. Implications for future research and treatment are discussed.
\end{abstract}

Keywords: skin picking, personality traits, emotion dysregulation, subtypes, MIDAS

\section{Introduction}

Pathological skin picking (SP) is a neuropsychiatric condition characterized by repetitive picking behaviors, which result in significant tissue damage and are accompanied by marked distress or functional impairment. ${ }^{1}$ While it was not categorized in the Diagnostic and Statistical Manual of Mental Disorders Fourth Edition Text Revision (DSM-IV-TR), text revision ${ }^{2}$ and listed as an Impulse Control Disorder-Not Otherwise Specified, recently, SP has been included in the "Obsessive compulsive and related disorders" chapter of the DSM-5. Individuals with SP tend to pick small irregularities or blemishes or skin lesions such as scars, calluses, pimples, or scabs resulting from previous picks. ${ }^{3}$ The most common picking areas are more easily accessible, such as the face, arms, upper body, hands, legs, and cuticles. ${ }^{4}$ Epidemiological studies have reported that SP represents a relatively frequent condition, occurring in $1.4 \%-5.4 \%$ individuals of the community, ${ }^{3,4}$ is more prevalent among females, ${ }^{5}$ and has its onset in adolescence. ${ }^{6}$

Research suggested the presence of different phenomenological dimensions of SP. In semistructured interviews of 34 adults with SP, 24\% reported full awareness of 
picking episodes, while $76 \%$ said that picking sometimes occurred out of awareness. ${ }^{7}$ Arnold et $\mathrm{al}^{8}$ conceptualized SP into three subtypes: a "compulsive" subtype, characterized by picking performed with full awareness and in response to aversive emotions; an "impulsive" subtype, characterized by picking performed with minimal awareness; and a "mixed" subtype, which shares both features. More recently, through online surveys on nonclinical samples, Walther et $\mathrm{al}^{9}$ identified an "automatic" SP subtype that tends to occur outside of one's awareness, including situations in which an individual picks his/her skin while engaged in a sedentary activity, such as reading or watching television, and a "focused" subtype, a more intentional behavior engaged in response to an urge or other negative emotion, or as an effort to correct perceived imperfections. Similarly, through factor analyses in large nonclinical and clinical samples, Pozza et $\mathrm{al}^{10}$ found an automatic, focused, and mixed SP subtype. The latter subtype was characterized by features of both automatic and the focused subtypes. SP has been conceptualized as belonging to the putative obsessive-compulsive spectrum, ${ }^{7}$ which assumes a relationship between compulsive and impulsive disorders. Some research focused on personality traits related to impulse control and emotion regulation. Impulsiveness, emotionality and emotional regulation difficulties, reward dependence, and harm avoidance have been reported among the personality traits in repetitive SP behaviors. ${ }^{3}$ According to the "Emotion Regulation Hypothesis" of SP, ${ }^{4}$ this behavior would have impulsive features: the individual experiences an irresistible impulse to pick skin, mounting tension or arousal prior to picking, and pleasure or gratification during the act. Snorrason et $\mathrm{al}^{3}$ investigated this hypothesis in undergraduates with or without history of pathological SP, reporting that SP was related to regulation difficulties and emotion reactivity. In addition, for most of those suffering from SP, intensity of negative affective feelings (ie, anxiety, tension, or boredom) was pronounced just before picking, and diminished significantly in the period from before to after picking. Relief and gratification increased during picking, whereas guilt increased afterward. Other authors studied comorbidity rates of personality disorders associated with SP; in a clinical sample, Keuthen et al ${ }^{11}$ found that pathological SP most commonly was associated with obsessive-compulsive personality disorder and borderline personality disorder.

Despite the recent inclusion in the DSM-5, pathological SP is a topic still understudied. Most studies investigated SP as a unitary condition, and the role of personality traits across SP subtypes has not been examined. Starting from these points, in a large community sample, the current cross-sectional study aimed to investigate which personality traits and emotion dysregulation deficits could predict specific SP subtypes.

\section{Methods}

\section{Participants}

A total of 289 adult community individuals were recruited. Of those, four were excluded since the MCMI-III validity scale scores resulted $>0$, suggesting an invalid protocol. Thus, the final study sample was composed by 285 community individuals (71.20\% women). Mean age was 34.98 years (standard deviation $=15.91$ ). All participants were white. Marital status was $61.10 \%$ single, $28.10 \%$ married or cohabitating, $10 \%$ separated or divorced, and $1.10 \%$ widowed. Forty-four percent of the sample were students, $88.10 \%$ had a full- or part-time job, 5.70\% unemployed, and 6.30\% retired. Participants were unscreened in order to obtain a more representative sample of the community population.

Data collection was carried out from November 2014 to August 2015. Through convenience sampling, participants were recruited in a variety of public settings in several cities located in the northern, mid, or southern Italy. Psychologists approached the participants in public settings, including high schools, universities, railway stations, libraries, malls, sports, or volunteering associations. When approached, each participant was provided with a brief overview of the study, particularly a description of SP behaviors. The fact that picking behaviors tend to also occur in the general population was highlighted. All the participants stated that they engaged in SP behaviors to some degree. If interested, he/she was taken aside to complete the questionnaires individually or in small groups. In accordance with the Ethical Principles of Psychologists and Code of Conduct, ${ }^{12}$ all the participants who were recruited, provided written informed consent to participate after having received a detailed description of the study aims. The study was approved by the ethics committee of the University of Florence. Participants' identities remained anonymous and participation was entirely volunteer and uncompensated. Contact information of the study coordinator (AP) was provided if participants had further questions or concerns regarding their participation. An overview of sociodemographic and clinical characteristics of the community sample is provided in Table 1 .

\section{Measures}

The Milwaukee Inventory for the Dimensions of Adult Skin Picking (MIDAS) ${ }^{9}$ is a 12-item self-report measure that assesses pathological SP. Each item of the MIDAS is rated from 1 (not true for any of my behaviors of skin picking) to 5 (true for all my behaviors of skin picking). The MIDAS is the only instrument designed to evaluate subtypes 
Table I Sociodemographic and clinical characteristics of the community sample $(n=285)$

\begin{tabular}{|c|c|c|}
\hline Characteristics & M (SD; range) & n (\%) \\
\hline Age & 34.98 (15.91; 18-84) & \\
\hline MIDAS automatic & $9.08(4.56 ; 5-25)$ & \\
\hline MIDAS focused & $6.09(3.17 ; 3-15)$ & \\
\hline MIDAS mixed & $6.60(3.14 ; 4-18)$ & \\
\hline Female & & $203(71.20)$ \\
\hline \multicolumn{3}{|l|}{ Marital status } \\
\hline Single & & $174(61.10)$ \\
\hline Married & & $80(28.10)$ \\
\hline Separated/divorced & & $28(10.00)$ \\
\hline Widowed & & $3(1.10)$ \\
\hline \multicolumn{3}{|l|}{ School license } \\
\hline Elementary school license & & $4(1.40)$ \\
\hline Middle school license & & $29(10.20)$ \\
\hline High school license & & $154(54.00)$ \\
\hline Degree or $\mathrm{PhD}$ & & $98(34.40)$ \\
\hline \multicolumn{3}{|l|}{ Employment status } \\
\hline Student & & $126(44.20)$ \\
\hline Employed & & $125(88.10)$ \\
\hline Unemployed & & $16(5.70)$ \\
\hline Retired & & $18(6.30)$ \\
\hline
\end{tabular}

Abbreviations: M, mean; MIDAS, Milwaukee Inventory for the Dimensions of Adult Skin Picking; SD, standard deviation.

of SP: a focused subtype, which typically concerns specific areas of the body and occurs in response to negative emotions (such as anger or anxiety), or bodily sensations, and an automatic subtype, which occurs without awareness during activities not related to the picking behaviors. The MIDAS items were modeled based on those of the Milwaukee Inventory for Subtypes of Trichotillomania-Adult Version (MIST-A), ${ }^{13}$ which measures the focused subtype of trichotillomania, occurring with some degree of awareness, and the automatic subtype. According to the authors of the measure, ${ }^{9}$ SP consists of two types of behaviors: those behaviors engaged by individuals who pick the skin in a targeted manner or in response to something and those behaviors engaged in automatically (out self-awareness). The hypothesis that there would be two different subtypes of SP, with phenomenological and functional characteristics similar to those of the subtypes of trichotillomania, is based on clinical observation and studies that suggest a strong association between SP and trichotillomania. ${ }^{14,15}$ The validation study ${ }^{9}$ was conducted through an online survey on a sample of 92 participants, who reported repetitive body-focused behaviors, including $\mathrm{SP}$ and trichotillomania. The validation study of the measure suggested three factors, assessing a focused, automatic, and mixed subtype, respectively.

The Millon Clinical Multiaxial Inventory-III (MCMI-III) ${ }^{16}$ is a 175-item self-report questionnaire, which was used to assess personality traits as a dimensional assessment tool. These are defined from raw scores of MCMI-III scales, weighted and converted to base rate (BR) scores. Fourteen clinical scales assess personality patterns related to DSM-IV disorders: eleven define personality pattern scales (schizoid, avoidant, depressive, dependent, histrionic, narcissistic, antisocial, sadistic, obsessive-compulsive, negativistic, and self-defeating), whereas three define severe personality scales (schizotypal, borderline, and paranoid). Among personality pattern scales, depressive, sadistic, and self-defeating can be considered more severe variants of avoidant, antisocial, and negativistic, respectively.

The Difficulties in Emotion Regulation Scale (DERS) ${ }^{17}$ is a 36-item self-report measure that assesses individuals' typical levels of emotion dysregulation across six domains, measured by six scales: nonacceptance of negative emotions, inability engaging in goal-directed behaviors when distressed, difficulties controlling impulsive behaviors when distressed, limited access to emotion regulation strategies perceived as effective, lack of emotional awareness, and lack of emotional clarity. Internal consistency in the current sample was very good across the scales (Cronbach's alpha ranging from 0.90 to 0.80 across the scales).

\section{Statistical analysis}

To investigate the relationship between SP subtypes, personality traits and emotion dysregulation, bivariate correlations were performed computing Pearson's $r$ coefficients between MIDAS, MCMI-III personality scale, and DERS scores. Power calculations were run for this analysis. For a medium effect size, $80 \%$ power, and significance set at $P<0.0002$, the required sample size for bivariate correlations was 201.

To test the contribution of personality traits to SP subtypes symptoms, stepwise multiple linear regression models were performed entering MCMI-III personality scale scores as predictors and MIDAS scores as outcomes. Power calculations were run for this analysis. For a medium effect size, $80 \%$ power, and significance set at $P<0.01$ (two tails), the required sample size was 82 .

Subsequently, stepwise multiple linear regression models were created by entering all MCMI-III personality scales and DERS scales scores as predictors and MIDAS scores as outcomes. Statistical analysis was conducted with SPSS software version 21.00 (IBM Corporation, Armonk, NY, USA).

\section{Results \\ Correlations between SP subtypes, personality traits, and emotion dysregulation}

MIDAS automatic scale scores positively moderately correlated with MCMI-III avoidant scale scores $(r=0.30$, 
$P<0.0002)$, masochistic $(r=0.31, P<0.0002)$, and borderline scale scores $(r=0.35, P<0.0002)$, positively weakly with depressive $(r=0.22, P<0.0002)$, dependent $(r=0.22, P<0.0002)$, negativistic $(r=0.28, P<0.0002)$, schizotypal scale scores $(r=0.24, P<0.0002)$. MIDAS automatic scale scores negatively weakly correlated with histrionic scale scores $(r=-0.20, P<0.0002)$. MIDAS focused scale scores positively weakly correlated with avoidant $(r=0.26, P<0.0002)$, depressive $(r=0.26, P<0.0002)$, dependent ( $r=0.25, P<0.0002)$, and schizotypal $(r=0.24$, $P<0.0002)$ and positively moderately correlated with negativistic $(r=0.33, P<0.0002)$, masochistic $(r=0.33$, $P<0.0002)$, and borderline $(r=0.38, P<0.0002)$. MIDAS focused scale scores negatively weakly correlated with obsessive-compulsive scale scores $(r=-0.20, P<0.0002)$. MIDAS mixed scale scores positively weakly correlated with sadistic scale scores $(r=0.21, P<0.0002)$. Bivariate correlation coefficients between MIDAS and MCMI-III scale scores are presented in Table 2.

MIDAS automatic scale scores positively weakly correlated with DERS inability to engage in goal-directed behaviors scores ( $r=0.29, P<0.0002)$, with DERS difficulties controlling impulsive behaviors $(r=0.26, P<0.0002)$ and DERS limited access to emotion regulation strategies ( $r=0.25, P<0.0002$ ). MIDAS focused scale scores positively and weakly correlated with DERS nonacceptance of negative emotions ( $r=0.25, P<0.0002$ ), DERS lack of emotional awareness $(r=0.29, P<0.0002)$, and DERS limited access to emotion regulation strategies $(r=0.27, P<0.0002)$. MIDAS

Table 2 Bivariate correlations coefficients between MCMI-III personality scales and MIDAS scales $(n=285)$

\begin{tabular}{llll}
\hline MCMI-III & $\begin{array}{l}\text { MIDAS } \\
\text { personality scales }\end{array}$ & $\begin{array}{l}\text { MIDAS } \\
\text { focused }\end{array}$ & $\begin{array}{l}\text { MIDAS } \\
\text { mixed }\end{array}$ \\
\hline Schizoid & 0.15 & 0.09 & 0.06 \\
Avoidant & $0.30^{*}$ & $0.26^{*}$ & 0.12 \\
Depressive & $0.22^{*}$ & $0.26^{*}$ & 0.07 \\
Dependent & $0.22^{*}$ & $0.25^{*}$ & 0.11 \\
Histrionic & $-0.20^{*}$ & -0.14 & -0.06 \\
Narcissistic & -0.12 & -0.15 & 0.05 \\
Antisocial & 0.15 & 0.17 & 0.12 \\
Sadistic & 0.18 & 0.19 & $0.21^{*}$ \\
Obsessive-compulsive & -0.16 & $-0.20^{*}$ & -0.14 \\
Negativistic & $0.28^{*}$ & $0.33^{*}$ & 0.19 \\
Masochistic & $0.3 I^{*}$ & $0.33^{*}$ & 0.10 \\
Schizotypal & $0.24^{*}$ & $0.24^{*}$ & 0.14 \\
Borderline & $0.35^{*}$ & $0.38^{*}$ & 0.17 \\
Paranoid & 0.08 & 0.17 & 0.10 \\
\hline
\end{tabular}

Note: $* P<0.0002$ ( $P$-value with Bonferroni correction).

Abbreviations: MCMI-III, Millon Clinical Multiaxial Inventory-III; MIDAS, Milwaukee Inventory for the Dimensions of Adult Skin Picking. focused moderately positively correlated with DERS inability to engage in goal-directed behaviors $(r=0.30, P<0.0002)$ and DERS difficulties in controlling impulsive behaviors $(r=0.31, P<0.0002)$. MIDAS mixed scale scores did not correlate with any DERS scale scores. Bivariate correlation coefficients between MIDAS and DERS scale scores are presented in Table 3.

\section{Personality traits and emotion dysregulation as predictors of SP subtypes}

Multiple linear regression analyses were conducted to investigate which personality traits predicted SP subtypes. Results showed that higher scores on the MCMI-III borderline $(\beta=0.32, t=4.47, P<0.001)$, avoidant $(\beta=0.18, t=2.59$, $P<0.01)$ significantly and uniquely predicted higher MIDAS automatic scale scores, after controlling for the effects of the other personality traits measured by the MCMI-III $\left(R^{2}=13.40 \%\right)$. Higher scores on the MCMI-III borderline scale $(\beta=0.38, t=6.75, P<0.001)$ significantly and uniquely predicted higher MIDAS focused scale scores, after controlling for the effects of the other personality traits measured by the MCMI-III $\left(R^{2}=14.70 \%\right)$. Higher scores on the MCMI-III sadistic scale $(\beta=0.21, t=3.57, P<0.001)$ significantly and uniquely predicted higher MIDAS mixed scale scores, after controlling for the effects of the other personality traits measured by the MCMI-III $\left(R^{2}=4.30 \%\right)$. Beta coefficients of MCMI-III scores on the MIDAS scores are provided in Table 4.

Subsequently, the effects of MCMI-III personality scales and DERS scores were tested on the MIDAS automatic scale entering all MCMI-III personality scales and DERS scales as predictors. Results showed that higher scores on

Table 3 Bivariate correlation coefficients between the MIDAS and DERS scores $(n=285)$

\begin{tabular}{llll}
\hline & $\begin{array}{l}\text { MIDAS } \\
\text { automatic }\end{array}$ & $\begin{array}{l}\text { MIDAS } \\
\text { focused }\end{array}$ & $\begin{array}{l}\text { MIDAS } \\
\text { mixed }\end{array}$ \\
\hline $\begin{array}{l}\text { DERS nonacceptance of } \\
\text { negative emotions }\end{array}$ & 0.19 & $0.25^{*}$ & 0.14 \\
$\begin{array}{l}\text { DERS inability engaging in } \\
\text { goal-directed behaviors }\end{array}$ & $0.29 *$ & $0.30^{*}$ & 0.18 \\
$\begin{array}{l}\text { DERS difficulties controlling } \\
\text { impulsive behaviors }\end{array}$ & $0.26^{*}$ & $0.31^{*}$ & 0.14 \\
$\begin{array}{l}\text { DERS lack of emotional } \\
\text { awareness }\end{array}$ & 0.19 & $0.29^{*}$ & 0.16 \\
$\begin{array}{l}\text { DERS limited access to } \\
\text { emotion regulation strategies }\end{array}$ & $0.25^{*}$ & $0.27^{*}$ & 0.17 \\
$\begin{array}{l}\text { DERS lack of emotional clarity } \\
\text { Note }\end{array}$ & 0.02 & -0.01 & -0.02 \\
\hline
\end{tabular}

Note: ${ }^{*} P<0.0002$ ( $P$-value with Bonferroni correction).

Abbreviations: DERS, Difficulties in Emotion Regulation Scale; MIDAS, Milwaukee Inventory for the Dimensions of Adult Skin Picking. 
Table 4 Linear regression analysis coefficients of MIDAS scores on MCMI-III personality scale scores $(n=285)$

\begin{tabular}{lllll}
\hline Outcomes & Predictors & $\boldsymbol{\beta}$ & $\boldsymbol{t}$ & $\boldsymbol{R}^{\mathbf{2}}(\%)$ \\
\hline MIDAS automatic & MCMI-III borderline & 0.32 & $4.47^{*}$ & 13.40 \\
& MCMI-III avoidant & 0.18 & $2.59^{* *}$ & \\
MIDAS focused & MCMI-III borderline & 0.38 & $6.75^{*}$ & 14.70 \\
MIDAS mixed & MCMI-III sadistic & 0.21 & $3.57^{*}$ & 4.30 \\
\hline
\end{tabular}

Note: $* P<0.001, * * P<0.01$.

Abbreviations: MCMIIIII, Millon Clinical Multiaxial Inventory-III; MIDAS, Milwaukee Inventory for the Dimensions of Adult Skin Picking.

the MCMI-III borderline scale $(\beta=0.28, t=4.88, P<0.001)$ and on the DERS difficulties engaging in goal-directed behavior $(\beta=0.19, t=3.27, P<0.01)$ predicted higher scores on the MIDAS automatic scale $\left(R^{2}=15.40 \%\right)$. The effects of the other MCMI-III personality and DERS scales were not significant.

The effects of MCMI-III personality scales and DERS were tested on the MIDAS focused scale. Results indicated that higher scores on the MCMI-III borderline ( $\beta=0.30$, $t=5.23, P<0.001)$ and DERS difficulties engaging in goaldirected behavior $(\beta=0.15, t=2.52, P<0.05)$ and DERS limited access to emotion regulation strategies $(\beta=0.21, t=3.26$, $P<0.01)$ predicted higher scores on the MIDAS focused scale $\left(R^{2}=22.30 \%\right)$. The effects of the other MCMI-III personality and DERS scales were not significant.

The effects of MCMI-III personality scales and DERS were tested on the MIDAS mixed scale. Results indicated that higher scores on the MCMI-III sadistic ( $\beta=0.19, \mathrm{t}=3.30$, $P<0.001)$ and DERS difficulties engaging in goal-directed behavior $(\beta=0.15, t=2.68, P<0.01)$ predicted higher scores on the MIDAS mixed scale $\left(R^{2}=6.90 \%\right)$. The effects of the other MCMI-III personality and DERS scales were not significant.

Beta coefficients of MCMI-III borderline, sadistic scales, DERS difficulties engaging in goal-directed behavior, and DERS limited access to emotion regulation strategies on MIDAS scales are presented in Table 5.

\section{Discussion}

Poor attention has been dedicated by researchers to pathological SP. Most studies investigated SP as a unitary condition, and the role of personality traits across SP subtypes has not been examined sufficiently. The current study investigated which personality traits and emotion regulation deficits could predict specific SP subtypes in a community sample.

Results suggested that automatic SP was moderately associated with avoidant, masochistic, and borderline traits and weakly associated with depressive, dependent,
Table 5 Linear regression analysis coefficients of the MIDAS scores on MCMI-III personality scales and DERS scores $(n=285)$

\begin{tabular}{|c|c|c|c|c|}
\hline Outcomes & Predictors & $\beta$ & $t$ & $R^{2}(\%)$ \\
\hline \multirow{2}{*}{$\begin{array}{l}\text { MIDAS } \\
\text { automatic }\end{array}$} & MCMI-III borderline & 0.28 & $4.88^{*}$ & 15.40 \\
\hline & $\begin{array}{l}\text { DERS difficulties engaging in } \\
\text { goal-directed behavior }\end{array}$ & 0.19 & $3.27 * *$ & \\
\hline \multirow{3}{*}{$\begin{array}{l}\text { MIDAS } \\
\text { focused }\end{array}$} & MCMI-III borderline & 0.30 & $5.23^{*}$ & 22.30 \\
\hline & $\begin{array}{l}\text { DERS difficulties engaging in } \\
\text { goal-directed behavior }\end{array}$ & 0.15 & $2.52 * * *$ & \\
\hline & $\begin{array}{l}\text { DERS limited access to } \\
\text { emotion regulation strategies }\end{array}$ & 0.21 & $3.26 * *$ & \\
\hline \multirow{2}{*}{$\begin{array}{l}\text { MIDAS } \\
\text { mixed }\end{array}$} & MCMI-III sadistic & 0.19 & $3.30 *$ & 6.90 \\
\hline & $\begin{array}{l}\text { DERS difficulties engaging in } \\
\text { goal-directed behavior }\end{array}$ & 0.15 & $2.68^{* *}$ & \\
\hline
\end{tabular}

Note: $* P<0.001, * * P<0.01$, $* * * P<0.05$.

Abbreviations: DERS, Difficulties in Emotion Regulation Scale; MCMI-III, Millon Clinical Multiaxial Inventory-III; MIDAS, Milwaukee Inventory for the Dimensions of Adult Skin Picking.

negativistic, and schizotypal traits. Individuals with higher levels of automatic SP had deficits with ability to engage in goal-directed behavior. Borderline and avoidant traits significantly and uniquely predicted automatic skin picking. These findings extended previous knowledge, which indicated that social anxiety disorder is one of the most common lifetime psychiatric disorders present among people with SP from the community. ${ }^{5}$ It could be believed that tissue damages produced by repetitive picking behaviors tend to increase fear of social interactions, thus reinforcing avoidant personality traits and maladaptive schemas through a vicious cycle. This appears consistent with the fact that individuals with more severe SP more frequently report avoiding social events or going out into public. The relation between avoidant traits and SP could be also viewed as moderated by poor body image and self-esteem. Thus, avoidance of social situations could serve as a maintenance behavior for individuals with SP and negative body image. Avoidant traits could be a specific type of personality traits involved in automatic SP but not in the focused one. Automatic SP behaviors could have the role of coping strategies for anxiety through reward dependence and harm avoidance mechanisms for people with avoidant personality, as highlighted by other authors. ${ }^{18}$ Given this potential role, it could be expected that psychotherapeutic work targeting avoidant personality traits could also produce positive effects on automatic SP behaviors. It could be interesting that future research test whether therapeutic interventions on avoidant personality are also associated with reduction in SP behaviors or vice versa. However, the cross-sectional nature of the study does not allow drawing 
firm conclusions about the causal connection between more severe picking behaviors and avoidant traits. Future studies should also examine whether avoidant traits moderate the relation between negative body image and SP behaviors.

The relation between deficits with ability to engage in goal-directed behavior, control impulsive behavior, and automatic SP extended previous data, which showed that higher levels of impulsivity are present in SP, and highlighted which specific emotion dysregulation deficits are associated with each specific SP subtype. As suggested by other authors in neuroimaging studies,${ }^{19}$ impulse control deficits could be explained by bilateral abnormalities in the anterior cingulate cortex and abnormalities on the left temporoparietal junction neural circuits involved in the generation and suppression of motor responses.

Overall, an interesting finding was that borderline traits were predictors of both automatic and focused SP behaviors, suggesting that this clinical picture could be associated with SP occurring out of awareness and SP engaged in response to negative feelings.

Different from automatic SP, focused SP was predicted also by lack of access to emotion regulation strategies. This result seemed to confirm the hypothesis that focused SP could be a maladaptive strategy to manage negative emotion and that it could benefit from psychotherapeutic interventions aimed at improving awareness of negative emotions, such mentalization-based psychotherapy. ${ }^{20}$ Moreover, individuals with SP and lack of strategies to manage negative emotions could benefit from psychotherapy delivered in a group setting, as shown by previous studies, which demonstrated that group psychotherapy has larger effects on alexithymia and emotion regulation relative to individual psychotherapy. ${ }^{21}$

It could be considered that the integumentary system is frequently the focus of tension-reducing and emotionregulating behaviors, especially during states of autonomic nervous system hyperarousal. ${ }^{22}$ This factor could be important in the pathogenesis of pathological SP, as suggested by other researchers. ${ }^{22}$ Mood-stabilizing drugs, such as lithium carbonate, that are used to treat disorders of emotional regulation have not been adequately studied in the management of the self-induced dermatoses and may prove to be very helpful in the management of SP. ${ }^{22}$

Mixed SP was uniquely predicted by sadistic traits but not by emotion dysregulation deficits. The relation between mixed SP and sadistic traits could be explained by trait impulsivity, since recent studies on community samples showed that trait impulsivity was a strong predictor of sadistic traits measured by the MCMI-III beyond the effects of emotion dysregulation. ${ }^{23}$ Consistent with another study conducted on a clinical sample showing a significant association between anger and sadistic traits assessed by the MCMI-III, ${ }^{24}$ mixed SP could be a maladaptive strategy of coping with anger in individuals with sadistic traits. The finding of an association between sadistic traits and mixed SP could have some useful implications for psychotherapeutic interventions, suggesting the use of assertiveness skills training techniques and rationalemotive therapy, which have been found to be an effective treatment strategy for trait anger, impulsivity, and sadistic characteristics. ${ }^{25}$ As highlighted by other researchers, ${ }^{25}$ people with sadistic traits and trait anger tend to exhibit distorted processing styles including selective abstraction, arbitrary inference, personalization, magnification, and minimization. They often report underlying beliefs about the fundamental unfairness of the world and condemnation of others.

Finally, some limitations should be considered. First, the sample used consisted of nonclinical individuals; thus, further studies with clinical samples with SP should be replicated. Consistently, another suggestion could be the assessment of categorical diagnoses of personality disorders, and not only the presence of personality traits, in patients with SP. Another suggestion for the future could be the assessment of specific traits related to borderline, avoidant, and sadistic personality with the aim to verify which traits are more closely associated with SP (eg, self-injurious behaviors or fear of abandonment for borderline patients and trait impulsivity for sadistic patients could be specific predictors of SP). On the contrary, some research has indicated that children who have experienced severe maltreatment and other adverse childhood events are more likely to engage in non-suicidal self-injury (NSSI), including SP. ${ }^{26}$ Thus, it could be argued that instead of SP being the reason for social avoidance, perhaps childhood trauma was predictive of both social avoidance and SP. One of the interesting findings regarding NSSI, ${ }^{27}$ although not specifically SP, is that it is related to state and affect dysregulation, with persons engaging in NSSI stating that it helps them move from a hyperactivated state, or a dissociative/numb state, to a feeling of normality. Therefore, it could be that the reason those individuals with borderline traits and more serious dysregulation engage in $\mathrm{SP}$ is, paradoxically, to feel more normal. Thus, a limitation of the current study could be that it did not assess the role of childhood adverse events as predictors of SP. Future research should focus on this point in order to investigate the role of adverse childhood experiences.

In conclusion, knowledge on clinical characteristics of SP subtypes, particularly personality traits, is relevant for 
clinical practice and should be extended in order to enhance the development of effective interventions for a still underrecognized neuropsychiatric condition, such as pathological SP behaviors.

\section{Disclosure}

The authors report no conflicts of interest in this work.

\section{References}

1. American Psychiatric Association. Diagnostic and Statistical Manual of Mental Disorders (DSM-5). Arlington, VA: American Psychiatric Publishing; 2013.

2. American Psychiatric Association. Diagnostic and Statistical ManualText Revision (DSM-IV-TR). Arlington, VA: American Psychiatric Publishing; 2000.

3. Snorrason I, Smári J, Ólafsson RP. Emotion regulation in pathological skin picking: Findings from a non-treatment seeking sample. J Behav Ther Exp Psychiatry. 2010;41(3):238-245.

4. Roberts S, O'Connor K, Bélanger C. Emotion regulation and other psychological models for body-focused repetitive behaviors. Clin Psychol Rev. 2013;33(6):745-762.

5. Odlaug BL, Lust K, Schreiber LRN, Christenson G, Derbyshire K, Grant JE. Skin picking disorder in university students: health correlates and gender differences. Gen Hosp Psychiatry. 2013;35(2):168-173.

6. Grant JE, Stein DJ, Woods DW, Keuthen NJ. Trichotillomania, Skin Picking, and Other Body-Focused Repetitive Behaviors. Arlington, VA: Psychiatric Publishing; 2012.

7. Arnold LM, McElRoy SL, Mutasim DF, Dwight MM, Lamerson CL, Morris EM. Characteristics of 34 adults with psychogenic excoriation. J Clin Psychiatry. 1998;59(10):1-478.

8. Arnold LM, Auchenbach MB, McElroy SL. Psychogenic excoriation. CNS Drugs. 2001;15(5):351-359.

9. Walther MR, Flessner CA, Conelea CA, Woods DW. The Milwaukee Inventory for the Dimensions of Adult Skin Picking (MIDAS): initial development and psychometric properties. J Behav Ther Exp Psychiatry. 2009;40(1):127-135.

10. Pozza A, Mazzoni GP, Patassini I, et al. Psychometric properties of the Italian version of the Milwaukee Inventory for the Dimensions of Adult Skin Picking (MIDAS) in clinical and non-clinical samples. Ital J Cogn Behav Psychother. 2016;22(1):35-63.

11. Keuthen NJ, Deckersbach G, Wilhelm S, et al. Repetitive skin-picking in a student population and comparison with a sample of self-injurious skin-pickers. Psychosomatics. 2000;41(3):210-215.

12. American Psychological Association. Ethical Principles of Psychologist and Code of Conduct. Washington, DC: American Psychological Association; 1992.
13. Flessner CA, Woods DW, Franklin ME, Cashin SE, Keuthen NJ, Board TLCSA. The Milwaukee inventory for subtypes of trichotillomaniaadult version (MIST-A): development of an instrument for the assessment of "focused" and "automatic" hair pulling. J Psychopathol Behav. 2008;30(1):20-30.

14. Lochner C, Simeon D, Niehaus DJ, Stein DJ. Trichotillomania and skin-picking: A phenomenological comparison. Depress Anxiety. 2002; 15(2):83-86.

15. Wilhelm S, Keuthen NJ, Deckersbach T, et al. Self-injurious skin picking: Clinical characteristics and comorbidity. J Clin Psychiatry. 1999;60(7):454-459.

16. Millon T. Millon Clinical Multiaxial Inventory-III: Manual, Person Assessments. 2nd ed. Minneapolis, MN: National Computer Systems; 1997.

17. Gratz KL, Roemer L. Multidimensional assessment of emotion regulation and dysregulation: Development, factor structure, and initial validation of the difficulties in emotion regulation scale. J Psychopathol Behav. 2004;26(1):41-54.

18. Shusterman A, Feld L, Baer L, Keuthen N. Affective regulation in trichotillomania: evidence from a large-scale internet survey. Behav Res Ther. 2009;47(8):637-644.

19. Grant JE, Odlaug BL, Hampshire A, Schreiber LRN, Chamberlain SR. White matter abnormalities in skin picking disorder: a diffusion tensor imaging study. Neuropsychopharmacology. 2013;38(5):763-769.

20. Bateman A, Fonagy P. Mentalization-Based Treatment for Borderline Personality Disorder: A Practical Guide. Oxford: Oxford University Press; 2006.

21. Rufer M, Hand I, Braatz A, Alsleben H, Fricke S, Peter H. A prospective study of alexithymia in obsessive-compulsive patients treated with multimodal cognitive-behavioral therapy. Psychother Psychosom. 2004; 73(2):101-106.

22. Gupta MA. Emotional regulation, dissociation, and the self-induced dermatoses: clinical features and implications for treatment with mood stabilizers. Clin Dermatol. 2013;31(1):110-117.

23. Garofalo C, Velotti P, Zavattini GC. Emotion dysregulation and psychopathology: the case of personality disorders not otherwise specified. Eur Psychiatry. 2015;30(1):412.

24. DiGiuseppe R, Tafrate RC. Anger Disorders Scale: Manual. Toronto, Ontario, Canada: Multi Health Systems; 2004.

25. McDermut W, Fuller JR, DiGiuseppe R, Chelminski I, Zimmerman M. Trait anger and Axis I disorders: Implications for REBT. J Ration Emot Cogn Behav Ther. 2009;27(2):121-135.

26. Kaess M, Parzer P, Mattern M, et al. Adverse childhood experiences and their impact on frequency, severity, and the individual function of nonsuicidal self-injury in youth. Psychiatry Res. 2013;206(2):265-272.

27. Lloyd-Richardson EE, Perrine N, Dierker L, Kelley ML. Characteristics and functions of non-suicidal self-injury in a community sample of adolescents. Psychol Med. 2007;37(8):1183-1192.
Neuropsychiatric Disease and Treatment

\section{Publish your work in this journal}

Neuropsychiatric Disease and Treatment is an international, peerreviewed journal of clinical therapeutics and pharmacology focusing on concise rapid reporting of clinical or pre-clinical studies on a range of neuropsychiatric and neurological disorders. This journal is indexed on PubMed Central, the 'PsycINFO' database and CAS,

\section{Dovepress}

and is the official journal of The International Neuropsychiatric Association (INA). The manuscript management system is completely online and includes a very quick and fair peer-review system, which is all easy to use. Visit http://www.dovepress.com/testimonials.php to read real quotes from published authors. 